\title{
El campo educativo: Ensayo sociológico sobre su diferenciación y complejización creciente en Chile y América Latina
}

\author{
Educational Field: A sociological Essay about its increasing differentiation and \\ complexity in Chile and Latin America
O campo educativo: ensaio sociológico sobre sua diferenciação e complexização no Chile e América Latina

\author{
Javier Corvalán $R$. \\ Universidad Alberto Hurtado, Facultad de Educación. Erasmo Escala 1825, Santiago. \\ E-mail: jcorvala@cide.cl
}

\begin{abstract}
RESUMEN
El artículo analiza la creciente diferenciación y complejización que ha tenido en campo educativo en Chile y en América Latina a partir de la teorización que de tal concepto ha realizado la sociología de Pierre Bourdieu. Se asume la constitución del campo educativo como un espacio social de disputa permanente por legitimidad entre agentes dotados de capitales diversos argumentando que, particularmente en las últimas décadas, el campo en cuestión ha tendido a densificarse y a cambiar el posicionamiento de los agentes, incorporando también a nuevos agentes en torno a la figura de economistas de la educación, bajo el capital cultural y simbólico que provee la teoría del capital humano.
\end{abstract}

Palabras clave: campo educativo, espacio social, capital, agentes.

\begin{abstract}
This article analyzes the increasing differentiation and complexity of the educational field in Chile and in Latin America starting from the theorization that Pierre Bourdieu' sociology has made of it. The make-up of the educational field is thought of as a social space of permanent quarrel for legitimacy among agents who have a variety of capitals. It is argued that this field, particularly in recent decades, has become more intricate and led to a shift in the agents' position, allowing for the incorporation of new ones based on educational economists, who function under the cultural and symbolic capital provided by human capital theory.
\end{abstract}

Key words: Educational field, social space, capital, agents.

\section{RESUMO}

Analisa-se a crescente diferenciação e complexização que o campo educativo no Chile e América Latina tem sofrido a partir da teorização feita de tal conceito pela sociologia de Pierre Bordieu. Assume-se a constituição do campo educativo como um espaço social de disputa permanente por legitimidade entre agentes dotados de recursos diversos; argumenta-se que, particularmente, nas últimas décadas, este campo tem tendido a densificação em torno da figura de economistas da educação e da mudança de posicionamento dos agentes, incorporando também outros agentes na figura de economistas da educação, submetida ao capital cultural e simbólico e provida pela teoria do capital humano.

Palavras chave: campo educativo, espaço social, capital, agentes.

* Trabajo realizado con el auspicio del Centro de Estudios de Políticas y Prácticas en Educación (CEPPE), financiado por CONICYT-Chile. Agradezco los comentarios a una versión preliminar de este artículo de Alejandra Falabella, Rosa Gaete y Sara Joiko, académicas de la Facultad de Educación de la Universidad Alberto Hurtado. Por supuesto, las insuficiencias del texto son responsabilidad del autor. 
"Tendrá el Senado especialísimo cuidado de fomentar en la capital y en todas las ciudades y villas, el establecimiento de escuelas públicas e institutos o colegios, donde sea formado el espíritu de la juventud por los principios de la religión y de las ciencias"

(Artículo $8^{\circ}$ del Proyecto de Constitución Provisoria para el Estado de Chile publicado e 10 de Agosto de 1818 y sancionado y jurado solemnemente el 23 de octubre del mismo año).

"La instrucción dada en las escuelas primarias tendrá por objeto la educación física, la educación moral y la educación intelectual del menor" (Artículo 16, Ley No 3.654 sobre Educación Primaria Obligatoria publicada el 26 de agosto de 1920)

"Una educación que fomente una escala de valores morales y espirituales propios de nuestra tradición chilena y cristiana, que respecte la libertad de enseñanza y el fuero íntimo de la conciencia de cada cual, y que alcance a todos, tendrá que robustecer el conocimiento y amor de cada uno de nuestros compatriotas hacia Chile, su geografía, su historia y su pueblo. Del reencuentro de las raíces con la nacionalidad surgirán valores y virtudes que mucho pueden aportar en el difícil desafio que afrontamos"

(Declaración de Principios del Gobierno de Chile, 11 de marzo de 1974).

"La educación tiene por objeto el pleno desarrollo de la persona en las distintas etapas de la vida" (Artículo 10, Constitución Política de la República de Chile de 1980)

"La educación es el proceso permanente que abarca las distintas etapas de la vida de las personas y que tiene como finalidad alcanzar su desarrollo moral, intelectual, artístico, espiritual y físico mediante la transmisión y el cultivo de valores, conocimientos y destrezas, enmarcados en nuestra identidad nacional, capacitándolas para convivir y participar en forma responsable y activa en la comunidad" (Artículo 2,

Ley Orgánica Constitucional de Enseñanza, 10 de marzo de 1990)

"La educación es el proceso permanente que abarca las distintas etapas de la vida de las personas y que tiene como finalidad alcanzar su desarrollo moral, intelectual, artístico, espiritual y físico mediante la transmisión y el cultivo de valores,

conocimientos y destrezas. Se enmarca en el respeto y valoración de los derechos humanos y de las libertades fundamentales, de la diversidad multicultural y de la paz, y de nuestra identidad nacional, capacitando a las personas para convivir y participar en forma responsable, tolerante, solidaria democrática y activa en la comunidad y para trabajar y contribuir al desarrollo del país" (Artículo 2, Ley General de Educación, 2009).

"La educación es un bien de consumo"

(Alocución libre de Sebastián Piñera, Presidente de Chile, 19 de julio de 2011).

La noción de campo, entendida como escenario de disputas sociales de naturaleza histórico-simbólicas en el cual se despliegan capitales diversos así como también disposiciones estructurales proviene, principalmente, de la sociología de Pierre Bourdieu. Desde tal marco de análisis este concepto debe relacionarse con: i) una definición inicialmente útil a una teoría social contemporánea de tipo general y posteriormente adoptada para el análisis de fenómenos sociales específicos tales como la religión (Bourdieu, 1971), la creación literaria (Bourdieu, 1998), la política (Bourdieu, 2000a) y el mercado de bienes inmuebles (Bourdieu, 2000b), entre otros; ii) una interpretación de la sociedad como un espacio de relaciones de fuerza entre diferentes agentes, disputa que es principalmente 
simbólica en torno a un principio de legitimidad de aquello que se propone como el objeto mismo en disputa. Con esto, se parte de la base que las sociedades actuales han generado ciertos fenómenos de aparente consenso -entre ellos la educación- pero que los mismos están permanentemente sometidos al debate respecto de su sentido y legitimidad; iii) consecuentemente con lo anterior, la noción de campo se refiere también a un análisis del poder en estas sociedades a partir de una disputa por la definición del sentido, propósito u orientación de una determinada esfera o área de particularidad social, a partir de los interés de sus agentes o participantes.

De esta manera, tanto la teoría sociológica global de Bourdieu como lo específico de la misma cuando alude al concepto de campo, se enmarca dentro de las sociologías de la diferenciación social (Martuccelli, 1999), que entienden a la modernidad como un movimiento permanente de generación de espacios sociales particulares, los que de manera progresiva se vuelven mutuamente ininteligibles, aumentando su complejidad y su especialización. A partir de estos elementos iniciales haremos a continuación un breve desarrollo del concepto de campo en tanto propuesta explicativa de fenómenos relevantes de las sociedades contemporáneas para, posteriormente, proponer su vinculación con el análisis de escenarios educativos.

La noción que Bourdieu desarrolla respecto a campo surge de una crítica, tanto implícita como explícita, a ciertos marcos clásicos de análisis sociológico de los años 60 , a saber el estructural funcionalismo ${ }^{1}$ por una parte y el marxismo estructural ${ }^{2}$, por otra. Por un lado, la crítica al primero dice relación con una visión armónica y en equilibrio que tal corriente teórica tiene de la sociedad contemporánea y, en lo que respecta a los sistemas educativos, como proveedores de oportunidades de movilidad social a los individuos y de cohesión y capacidad productiva a las sociedades (Parsons, 1988). Si es posible hablar de campo educativo a partir de tal corriente teórica, este estaría marcado por el consenso entre los agentes que en él participan en torno a las necesidades que el sistema educativo satisface para la sociedad -principalmente integración social y movilidad social meritocrática-, todo ello a partir de un requisito, en términos de ideal normativo, de igualdad de oportunidades educativas. Desde el enfoque estructural-funcionalista entonces, la educación y más específicamente el campo educativo es un elemento más, entre otros, que intenta regular la sociedad, buscando armonizar las expectativas individuales con las necesidades del colectivo (Parsons, 1988).

Por otro lado, la crítica de la perspectiva de Bourdieu al marxismo estructural se refiere a una visión rígida de la distribución del poder a partir de la organización de clases sociales y de sus correlatos económicos. Cualquier análisis de campo o escenario educativo que se haga desde esta perspectiva tendrá, en la crítica de Bourdieu, el error de considerar a tales escenarios como un reflejo directo de la estructura de clases y del conflicto inherente en ellas. ${ }^{3}$ En tal perspectiva, el campo educativo no es sino un reflejo directo de la dominación económica, sin mayores innovaciones en su interior y

Representado principalmente por los aportes de Parsons (1988).

Ejemplificado por la visión que de las sociedades capitalistas contemporáneas muestra Althusser (1988).

Es la perspectiva que Althusser expone al considerar al sistema educativo, tanto como al sistema político, religioso, estatal, etc., como "aparatos ideológicos del Estado" destinados a mantener la dominación de clase en la sociedad. 
no habiendo en él una autonomización o diferenciación relevante respecto de la lógica de organización global de la sociedad.

En la noción de campo desarrollada por Bourdieu, ninguno de los enfoques anteriores da real cuenta de la complejidad de las sociedades actuales en las cuales lo simbólico se entremezcla con lo económico con el fin de reproducir el status quo imperante, pero bajo procesos de diferenciación constantes y crecientes. Tales procesos suceden al interior de campos, es decir, de realidades analíticas solamente comprensibles a partir de esta dinámica de diferenciación y, si bien es cierto cada campo posee un funcionamiento formalmente similar a los restantes, estos son sustantivamente distintos entre sí ya que cada uno obedece a una génesis e intereses históricos particulares. Así por ejemplo, el campo educativo tiene una estructura de funcionamiento similar al político o al artístico, pero son sus elementos sustantivos y su génesis y desarrollo histórico los que explican su dinámica particular, distinta y diferenciada.

De acuerdo a lo dicho en el párrafo anterior, es muy difícil entender el aporte que Bourdieu hace al análisis social contemporáneo, si su conceptualización se enmarca dentro de una reflexión mayor vinculada a la comprensión de este proceso creciente de diferenciación y especialización de las sociedades contemporáneas. A partir de tales procesos, ciertos criterios tradicionales de explicación de la identidad y las orientaciones de acción de individuos y grupos tales como clase, nación o adscripción ideológica, religiosa o política serían solo parcialmente explicativos de la producción discursiva y simbólica de los mismos y, en contrapartida, deberían ser reemplazados o complementados por el desenvolvimiento de agentes, encarnados finalmente en individuos, al interior de campos diversos con funcionamientos y orientaciones altamente estructurados, en los cuales solo ciertas orientaciones y códigos son legítimos, inteligibles y dotados de poder.

Para entrar en la explicación de la complejidad actual Bourdieu desarrolla entonces esta noción de campo que en sus propias palabras es un microcosmos autónomo al interior de un macrocosmos social (Bourdieu, $\mathrm{P}, 2000^{\mathrm{a}}$ p. 52). Dicho de otra manera, si existe un campo educativo en la sociedad éste es identificable en principio porque tiene una propia lógica de validación de los argumentos que le atañen:

La evolución de las sociedades tiende a hacer aparecer universos (los que denomino campos), que tienen leyes propias, que son autónomos...tenemos así universos sociales que tienen una ley fundamental un nomos independientes del aquel de otros universos que son auto-nomos que evalúan lo que hacen, los desafíos que en ellos se producen según principios y criterios irreductibles a aquellos de otros universos (Bourdieu, 1994: 19).

En esta perspectiva teórica es importante señalar que los agentes, reflejados en última instancia en individuos, transitan en su vida cotidiana entre diferentes campos y que, para desempeñarse en cada uno de ellos, necesitan códigos, lenguaje y capital que los habilite y les otorgue un determinado nivel de legitimidad como participantes. Este aspecto lo sintetiza Bourdieu con otros dos conceptos centrales en su teorización, el habitus por una parte y la disposición de capitales en su expresión económica, simbólica cultural y social, por otra. El habitus hace referencia a una práctica interiorizada que se transforma en una fuerza motora del pensamiento y de la acción y que produce disposiciones y prácticas similares entre los individuos (Bourdieu, 1982), en tanto los capitales reflejan un acumulado de valor disponible mediante el cual los agentes del campo adquiere legitimidad posicional en él: 
En cada momento el estado de las relaciones de fuerza entre los jugadores define la estructura del campo: se puede imaginar que cada jugador tiene ante sí pilas de fichas de diferentes colores, correspondientes a las diferentes especies de capital que detentan, de modo que su fuerza relativa en el juego, su posición en el espacio de juego y también sus estrategias de juego - lo que en francés se llama su «juego», las jugadas más o menos arriesgadas, más o menos prudentes, más o menos subversivas o conservadoras - dependen a la vez del volumen global de sus fichas y de la estructura de sus pilas de fichas, es decir, del volumen global y de la estructura de su capital (Bourdieu, 1992: 74-75).

Para el tema que nos interesa aquí podemos decir que nuestro postulado teórico central es que en el caso del campo educativo se genera, regenera y reproduce una determinada manera de referirse y analizar la problemática educativa así como de aquello que se va a considerar como educativo propiamente tal, y que tales distinciones no serán claramente comprensibles ni explicables desde su exterior, es decir, desde otros campos. En esta perspectiva, podemos pensar que si existe un campo educativo propiamente tal, en él se produce no sólo la discusión educativa sino también la validación de aquello que es legítimamente discutible y cuestionable en tanto educación. Esto significa también que al interior del campo educativo se produce no solo la legitimidad sino que, con ella se estructura también la jerarquización de quienes participan en la disputa posicional. Como señalamos, el campo entonces está definido y a la vez da cuenta de esta jerarquía de quienes se encuentran en su interior, y con ello da cuenta también de la distribución de posiciones en el espacio social. Los agentes que participan en el campo lo hacen a partir de sus disposiciones (habitus), pero también a partir de sus recursos, es decir, su volumen de capital.

La idea de agente, en tanto participante del campo, dice relación con el rol de transmisor y operador de habitus y capital y no de recreador del mismo y eventual innovador, lo que correspondería a un actor propiamente tal (tal como lo propone la sociología accionalista) Precisamente esta pasividad del agente o institución limitado a tener el desempeño que la estructura del campo exige, ha sido uno de los puntos más criticados de la concepción de Bourdieu y refleja, además, uno de los debates teóricos mas importantes de la sociología contemporánea.

Esta visión de los campos en los cuales se lleva a cabo la disputa de un objeto social y del espacio de diferenciación y jerarquización, nos lleva a la noción central también en el planteamiento de Bourdieu, de autonomía relativa (Bourdieu, 1992). Por tal este autor refiere, precisamente, a la capacidad que tiene el campo de autonomizarse, es decir, el hecho de formar un referente independiente -al menos relativamente- de lo que ocurre en los restantes campos sociales. La autonomización debe entenderse de manera distinta a la diferenciación puesto que esta última se refiere a la constitución de tantos campos como intereses y, por lo tanto, ámbitos de disputa, existan, mientras que la autonomía dice relación con procesos específicos, tales como tipos de ordenamiento o de jerarquización, que ocurren en su interior que pueden ser no solo distintos sino incluso contradictorios con lo que ocurre en los restantes campos.

Por ejemplo, es pensable que el campo de las finanzas ordena a los agentes jerárquicamente de acuerdo a su disposición de capital económico y de otros capitales como el cultural y social que permiten o posibilitan realizar nuevas y mejores inversiones que aumenten el capital económico inicial. En tal caso, el habitus dominante probablemente estará relacionado con la legitimidad, orientación y disposición al lucro, expresado 
cuantitativamente en la capacidad de aumentar los volúmenes de capital, es decir, en la explicación del cómo y del porqué del interés del campo y del juego de los agentes en él, organizado en torno al aumento constante del capital económico. El campo educativo, en contrapartida, jerarquiza a los agentes priorizando la disposición de otros capitales, dentro de los cuales destaca el de tipo cultural dado que el habitus que estructura este espacio social refiere a la disposición de conocimiento y a su certificación o capital cultural institucionalizado (Bourdieu, 1997). Él o los códigos de base del campo educativo subordinan, e incluso, devalúan, aun cuando no anulan por completo, la importancia del capital económico como elemento distintivo central y/o garantizador de posiciones en el espacio social.

Así, el campo educativo se autonomiza de lo que sucede en otros campos a pesar de funcionar al interior de una sociedad capitalista, lo que parece estar más armónicamente vinculado, por ejemplo, con el campo de las finanzas, pero esta autonomización es también relativa por cuanto la jerarquía de los agentes tiene cierta correlación con su disposiciones de capital económico, ya que los mismos, particularmente en sociedades capitalistas, no funcionan totalmente independizados del capital cultural y del capital social.

De esta manera, la tendencia a la autonomización de cada campo se manifiesta, entre otros elementos, en el lenguaje y los códigos autorreferenciales de los mismos que hacen que determinados agentes adquieran o disminuyan su poder y legitimidad, y en las formas de capital y tipo de habitus que son propios del campo. Es precisamente la cantidad y forma de capital que los agentes posean al interior del campo, así como el grado de internalización del habitus respectivo, los que generan jerarquía posicional al interior del mismo. Este último aspecto es de gran relevancia en la construcción teórica de Bourdieu y se refiere a que, como consecuencia de lo dicho anteriormente, todo campo se encuentra controlado, al menos parcialmente, por algunos de sus agentes. Éstos, a su vez, ejercen tal control de manera relacional, es decir, para constituirse jerárquicamente requieren de la existencia de otros agentes, que estos últimos luchen por adquirir una mejor posición y que, al hacerlo, reconozcan implícitamente su jerarquía inferior en relación a quienes ostentan una superior.

Para analizar el campo educativo es necesario entonces conceptualizar tanto el tipo de habitus dominante y válido en el mismo, como también las formas y tipos de capitales que sirven para actuar e imponer posiciones en él, aspectos que son comprensibles a partir de la aceptación de tal campo como espacio autónomo y diferenciado de otros campos.

Pese a lo anterior, es difícil pensar que el campo educativo se encuentre totalmente autonomizado de la influencia de otros campos ya que, tal como lo muestra la historia de las sociedades occidentales y, en particular, de las latinoamericanas durante el siglo $\mathrm{XX}$, es clara la influencia que la educación recibe primero del campo político y cultural y luego del económico (Archer, 1984). Esto se refleja, como dijimos a nivel del uso del lenguaje para estructurar la legitimidad de la educación, así como del debate y la jerarquía de quienes han participado en el campo educativo. En efecto, el campo educativo consolidado durante los siglos XIX y XX en la mayor parte de las sociedades de tradición o desarrollo occidental es un claro ejemplo de diferenciación y complejización constante, lo que comprende dinámicas aceleradas de reordenamiento en su interior.

Es así como en el caso de América Latina ciertos fenómenos comunes ocurridos en la historia de sus países llevaron a estructurar elementos también comunes en el campo educativo. En esta línea y como se sabe, el hecho histórico-político más notable en las 
primeras dos décadas del siglo XIX en América Latina es la independencia de la corona española y su constitución como repúblicas autónomas. En tal proceso, la mayor parte de estas repúblicas intenta consolidar un sistema educacional que edifique y construya la nación como sentimiento de pertenencia colectiva (Anderson, 1993), lo que se produce en sociedades que todavía no han diferenciado totalmente el campo civil y público de la esfera religiosa; dicho en otras palabras, no hay en tal momento histórico un terreno claro para la plena constitución del laicismo y para que los agentes que lo encarnan dominen la discusión educativa. Este tema es claramente identificable en Chile y en otros países en la pugna liberal-conservadora y en el triunfo de la primera que lleva a fundar las bases de Estado-docente laico en la segunda mitad del siglo XIX y que se consagraría como esfera de autonomía relativa a comienzos de siglo XX (Barros Arana, 1902; Labarca, 1939; PIIE, 1983; Serrano, 1994; Núñez, 1997 y Egaña, 2000).

Estos procesos históricos pueden ser leídos en torno a cambios profundos en el campo educativo que implican a sus agentes, habitus, capitales y orden jerárquico en su interior. Es así como el campo educativo en los países de tradición occidental pasa de un dominio de los contenidos y agentes religiosos estableciendo que los fines de la educación son la producción de buenos cristianos (Durkheim, 1972; 1975), centrando así la discusión y disputa en su interior en la pedagogía (Durkheim, 1982) e identificada ésta con un concepto moral-religioso, a una secularización constante mediante la cual el objetivo es la formación del ciudadano observante y obediente de normas (por ejemplo Sarmiento, 1849, para el caso de Argentina y Amunátegui y Amunátegui, 1856, para el caso de Chile), ubicando al Estado en el vértice superior de la jerarquización del campo, centrando la disputa intra-campo en el equilibrio entre saberes ciudadanos y aprendizajes científico-humanistas, e identificando a la pedagogía con la construcción de normas y de una moral laica (Durkheim, 1972). Esta estructuración del campo educativo, que junto a otros elementos políticos posteriores va a marcar gran parte del desarrollo educativo latinoamericano del siglo XX, tiene un tercer reordenamiento histórico con el discurso de transformación productiva instalado en el continente, a partir del llamado consenso de Washington a comienzos de los años 90 y refrendado por un influyente documento de CEPAL y UNESCO (1992), en la misma época.

En particular tales agentes (CEPAL y UNESCO) reposicionan la jerarquía dominante de un tipo de referente del campo educativo consistente en la definición de lo educacional a partir de la teoría del capital humano. Es importante considerar a tal ejercicio como un reposicionamiento puesto que la mencionada teoría había sido ya propuesta en la década del 60 por Schultz (1963) y Becker (1966) sin demasiado éxito más allá de lo estrictamente disciplinario en economía. Esto hizo que, en consecuencia, durante al menos dos décadas, la teoría del capital humano quedara relegada al campo disciplinar de la economía, conceptualizándose solo como la lectura económica de la educación y no como la interpretación correcta o dominante del fenómeno educacional. Así, esta teoría y sus agentes portadores se traspasan desde un campo disciplinar hacia uno políticopúblico y educativo, tendiendo a dominarlo, es decir, a situarse en él desde una jerarquía posicional que re-definirá los términos y credenciales legítimamente válidos para entrar en el debate educacional.

La mencionada acción y producción simbólica de agentes de fuerte legitimidad social como los anteriormente mencionados en los años 90 (CEPAL, UNESCO, Banco Mundial, entre otros) generó entonces una inclusión dominante en el campo educativo 
de los referentes intelectuales de la teoría del capital humano, es decir, a los agentes incluidos en la categoría de economistas de la educación, fuertemente excluidos hasta ese momento de la discusión educacional, así como también, y por extensión se incorporó jerárquicamente a las instituciones académicas y no académicas vinculadas, inspiradas o propulsoras de tal enfoque y por añadidura al mundo productivo privado, ya que la visión de la teoría del capital humano hace que los resultados de la educación y, por lo tanto, la legitimidad normativa de su discusión se validen, necesariamente, en el campo productivo y de inserción laboral.

En definitiva, el concepto de campo sugerido por Bourdieu nos parece útil en varios de sus aspectos para analizar la realidad educacional actual en Chile y en consecuencia si se hace el ejercicio desde esta perspectiva, un análisis de este campo educativo debería al menos considerar lo siguiente:

a) La existencia misma de este campo determinado por la concurrencia de diversos agentes al debate y de la producción discursiva y simbólica sobre una temática social, en este caso la educación. Si esto es así, habría que reconocer que este campo se ha densificado en las últimas décadas debido a la incorporación de nuevos agentes, lo que ha significado un aumento del debate, complejización y especialización creciente de las temáticas tratadas en su interior. Esto último parece evidente cuando, por ejemplo, se observa que en la segunda mitad del siglo XX se consolidan académica y políticamente diferenciaciones y especializaciones dentro de las "ciencias de la educación", tales como el currículum, la pedagogía, la organización de los sistemas educativos, la formación docente, etc.

Es así también como hace tres o cuatro décadas los principales integrantes del campo educativo en América Latina eran básicamente el Estado, la iglesia y algunos agentes privados y sindicales (estos últimos generalmente unificados). Por lo menos cinco grandes procesos que han cambiado y complejizado este panorama. El primero de ellos es la creciente presencia de la educación privada tanto en términos cuantitativos como de legitimidad social. En efecto, en la mayoría de los países latinoamericanos existía desde los inicios del sistema educativo -aún con diferentes grados de presencia- un conjunto importante de oferentes privados de educación el cual ha aumentado su número y con ello su legitimidad en las conversaciones al interior del campo educativo.

En segundo lugar, los procesos de globalización y de internacionalización han implicado la constitución en este campo de los llamados organismos internacionales de tipo financistas, en particular el Banco Mundial y el Banco Interamericano de Desarrollo, los que participan del debate a partir de una visión que les es propia y que se vincula a la concepción de la educación a partir de la teoría del capital humano y que no necesariamente coincide con la de los restantes participantes.

En tercer lugar, el desarrollo de las ciencias sociales y en general la creciente legitimidad del mundo académico en lo que respecta a las temáticas de planificación educativa ha significado la irrupción de una diversidad de centros de estudios con influencia en la discusión educacional. Lo que se observa aquí es la incorporación del discurso académico-científico en el campo educativo representado tanto por la lógica experimental y de evidencia científica y, a la vez, por códigos de legitimidad basados en las credenciales académicas de tales agentes (capital cultural institucionalizado e incorporado, según los términos de Bourdieu, 1979). 
En cuarto lugar, los crecientes procesos de descentralización como aspecto clave de las discusiones educativas desde hace por lo menos dos décadas en América Latina han significado la presencia creciente en las conversaciones y disputas del campo educativo de los llamados actores locales e intermedios que siendo parte a veces de la estructura de gobierno y otras de la de Estado, no necesariamente son identificables con los posicionamientos del gobierno central en el campo educativo (Gvirtz, 2008). Esto último quiere decir que otra de las particularidades del campo educativo en las últimas décadas ha sido la diversificación del Estado como agente en el mismo. Respecto de este punto es importante recordar que los países latinoamericanos y en no pocos europeos, los sistemas educacionales surgieron y desarrollaron como proyectos estatales y nacionales de construcción de homogeneidad social y, por lo tanto, con discursos intrínsecamente opuestos a los poderes locales o regionales (Archer, 1984). Sin embargo, los códigos o referencias propias del campo educacional relativos al interés nacional por sobre los intereses particulares se han debilitado en las últimas décadas, lo que puede entenderse como un descenso en la generalidad (nación) y un aumento de la particularidad (localidad), lo que a la vez se contrapone de manera absoluta al aumento del discurso curricular de contenidos universales (estandarización de conocimientos necesarios como consecuencia de la globalización), reflejado en el peso creciente de las evaluaciones internacionales comparativas, que disminuyen el peso político de un particularismo curricular. Por esto se puede afirmar entonces que el campo educativo y el habitus de sus agentes han tenido en las últimas décadas un movimiento importante entre universalismo y particularismo, lo que en todo caso, incorpora nuevos elementos en disputa al campo en cuestión.

En quinto lugar y también vinculado a algunos de los procesos ya señalados, en las últimas décadas ha habido también un cambio en los tipos de agentes del campo educativo provenientes del ámbito académico. Nos referimos por tal a la creciente legitimidad, particularmente en países como Chile, de la categoría economistas dentro de la discusión educativa, lo que hipotéticamente se explica tanto por la presencia ya mencionada de los bancos de desarrollo como también por la creciente legitimidad de la racionalidad técnicoinstrumental para analizar las finalidades de la educación, tema que está representado por el protagonismo alcanzado por la mencionada teoría del capital humano como sinónimo del estudio científico y racional del fenómeno educativo. Es importante recordar que la mayor parte de los agentes participantes del campo educativo en el siglo XIX y en buena parte del siglo XX formaron parte de la categoría de humanistas y el discurso cientificista en su interior estuvo dominado por la presencia de los agentes de tipo pedagogos.

Al mismo tiempo, la presencia creciente esta categoría -economistas -se explica también -en especial en algunos países- por la también ya mencionada presencia incremental de actores privados en la oferta educativa y, en términos más específicos, pero también relacionados con este argumento, por los procesos de privatización y regulación mercantil de tal oferta.

b) Conjuntamente con los fenómenos señalados en los párrafos anteriores, el campo educacional ha tenido otro movimiento de diferenciación tendiendo paulatinamente a escindirse en nuevos campos, basado en los niveles de los sistemas educacionales. El ejemplo más claro al respecto que tiene más de medio siglo en los países más desarrollados y es bastante más reciente en América Latina se refiere a la diferenciación entre el campo de la educación escolar y el de la educación superior principalmente de tipo universitaria. En 
el caso de Chile, la desdiferenciación de ambos campos tiene una expresión institucional basada históricamente en la fundación de la Universidad de Chile, en 1842, a quien se le da no solo el monopolio de la educación universitaria, sino también la tutela de la educación escolar en el país, en especial de la educación secundaria (Serrano, 1994).

A partir de la segunda mitad del siglo XX, entonces, el campo de la educación superior comienza paulatinamente a separarse del campo educativo general y a constituirse como campo propio de manera definitiva, a nuestro parecer, hacia fines del siglo XX. Dentro del marco conceptual que aquí analizamos podemos considerar entonces que esta diferenciación y constitución de al menos dos campos diferenciados en educación se observa por cuanto sus elementos constitutivos, agentes en disputa, capitales dispuestos, jerarquía, principios de legitimación y habitus, son distintos en cada uno de ellos

c) La existencia de una producción simbólica propia del campo educativo, lo que requiere preguntarse si efectivamente tiene este campo un código y un habitus y a partir de ellos un tipo de debate y de legitimidad del mismo que sea de su exclusividad. Este es un tema complejo y que requiere de ciertas especificaciones, puesto que no se trata solamente de la presencia de cierta jerga propia del debate educacional sino, de manera mucho más profunda, de ciertas maneras legítimas de razonar y argumentar al interior del campo y también de ciertos elementos considerados verdades de base o aspectos no cuestionables de la comunicación presente en el campo. En el caso de la educación, cabría preguntarse aquí si estamos en presencia de un campo propiamente tal que tiene ciertos elementos considerados consensuales e irrenunciables al mismo -lo que no significa que no sean modificables- tales como la igualdad de oportunidades educativas o la gratuidad de la educación pública o el valor intrínseco del conocimiento y del aprendizaje, por ejemplo.

d) Otros dos aspectos nos parecen de primera importancia respecto de la producción simbólica de los agentes al interior del campo educativo y que dicen relación con su inevitable posicionamiento como grupos de interés o stakeholders, con la necesidad de ser visualizados -por los demás agentes- en términos societales, es decir, con intereses que se relacionan con el bien común y no sólo con su bienestar particular. Esto se explica por cuanto, a nuestro parecer, el principio democrático que rige como aspiración permanente de las sociedades actuales, implica que ningún agente puede presentarse en el debate público solo bajo la imagen de un grupo de interés, es decir, de un conglomerado cuya acción tiene como objetivo central la defensa de sus aspiraciones particulares. Si bien la representación como grupo de interés es inicialmente aceptable en una sociedad democrática, es la vez legítimamente insuficiente para tener una voz válida dentro de un campo de disputa pública y en particular dentro del campo educativo. Debido a esto, todos los agentes del campo educativo deben tener necesariamente un discurso público o societal entendiendo por ello una producción discursiva y simbólica que tenga relación con una noción de bien común pero que, a la vez, no se oponga a sus intereses en cuanto agentes.

En concreto, este principio de análisis respecto del campo educativo hace que los agentes que en él intervienen deban exponer sobre todo los intereses públicos o societales de sus propuestas conectándolas con sus beneficios privados, pero argumentando que estos últimos son consecuencia o requisito de los primeros. Un ejemplo de ello, tal vez el más claro, es la acción y propuesta sindical de los docentes, en las cuales sus 
reivindicaciones salariales son (o deben ser) planteadas como un requisito para la calidad educacional y no como un fin en sí mismo.

Este principio nos parece básico en un análisis de campo que se aplique a aquellos escenarios de tipo político-públicos en los cuales, por su enmarcamiento actual al interior de una sociedad de principios democráticos y por su utilización y disputa de recursos públicos al interior del mismo, generan un tipo de habitus y en general de código y de comunicación interna estructurado en torno a la negación o disminución del argumento de beneficio propio. Precisamente, la jerarquía y control en el campo educativo parece expresarse por la capacidad de los agentes dominantes para controlarlo a partir de una producción simbólica que trata de articular aspectos técnicos (eficacia educativa) y políticos (bien común a partir de una provisión educativa de determinadas características).

e) Por último, no es menor señalar también que la estructura del campo educativo tenga la particularidad -a diferencia de otros campos sociales- que los beneficiarios o destinatarios directos exhiban una escasa participación en la dinámica del campo mismo. En efecto, los niños asistentes a los niveles primarios tienen una participación de beneficiarios del proceso y solo en algunas ocasiones los jóvenes de nivel secundario se han constituido como actores del campo en cuestión, siendo uno de los casos recientes más notables en América Latina la movilización estudiantil del año 2006 en Chile, conocida como Revolución de los pingüinos (Donoso, 2010) y, más recientemente, por los eventos similares ocurridos el primer semestre de 2011 De esta manera, por lo general los actores y agentes del campo educativo expresan sus opiniones a nombre de otros, sin que estos otros sido mandatados por los primeros para tal fin. Estas constataciones ponen de manifiesto un aspecto central en el análisis del campo educativo actual en Chile y en América Latina y relativo al control del mismo y al tipo de capital para ejercerlo, lo que sugiere algunas preguntas finales ¿cuáles son actualmente estos capitales? ¿cuál es la jerarquía que se forma en el campo en cuestión? ¿cuál es habitus que es propio de este campo y que al mismo tiempo mantiene diferenciados y distanciados a los agentes y que los aúna en una misma manera de organizar el interés del campo?

\section{REFERENCIAS BIBLIOGRÁFICAS}

Althusser, L. (1988). Ideología y aparatos ideológicos de Estado. Buenos Aires: Nueva Visión. Amunategui, M.L. y Amunategui, G. V. (1856) De la Instrucción Primaria en Chile: lo que es y lo que debería ser. Santiago: Imprenta del Ferrocarril.

Anderson, B. (1993). Comunidades Imaginadas. Reflexiones sobre el origen y la difusión del nacionalismo. Buenos Aires: Fondo de Cultura Económica.

Archer, M. (1984). Social Origins of Educational Systems. Londres: Sage,

Barros Arana, D. (1902). Historia Jeneral de Chile. Santiago: Cervantes.

Becker, G. (1964). Human Capital. New York: National Bureau of Economic Research.

Bourdieu, P. (1971). Genèse et Structure du Champ Religieux. Revue Française de Sociologie, Vol XIII, 295-334.

Bourdieu, P. (1979), Les Trois Etapes du Capital Culturel. Actes de la Recherche en Sciences Sociales, Vol. XXX, p. 3-6.

Bourdieu, P. (1992). Réponses. París: Seuil.

Bourdieu, P. (1994) Raisons pratiques. Sur la théorie de l'action. Paris: Seuil 
Bourdieu. P. (1997). Capital cultural, escuela y espacio social. México: Siglo XXI.

Bourdieu, P. (1998). Les Règles de l'Art. Paris: Points.

Bourdieu, P. (2000a). Propos Sur le Champ Politique. Presses Universitaires de Lyon: Lyon.

Bourdieu, P. (2000b). Les Structures Sociales de l'Economie. Paris: Seuil.

CEPAl-Unesco (1992). Educación y conocimiento. Eje de la transformación productiva con equidad, Santiago: CEPAL-UNESCO.

Donoso, S. (2010). Dynamics of Change. The chilean pinguino movement and its impact on the educational agenda. Master Thesis, Oxford University.

Durkheim, E. (1972), La Educación Moral. Buenos Aires: Shapire.

Durkheim, E. (1975), Educación y Sociología. Barcelona: Península.

Durkheim, E. (1982). Historia de la Educación y de las Doctrinas Pedagógicas. La Evolución Pedagógica en Francia. Madrid: La Piqueta.

Egaña.M. L. (2000). La Educación Primaria Popular en el Siglo XIX en Chile: una práctica de política estatal. Santiago: Lom.

Gvirtz, S. (2008). Equidad y Niveles Intermedios de Gobierno en los Sistemas Educativos. Buenos Aires: Aique Grupo Editor.

Labarca, A. (1939). Historia de la Enseñanza en Chile. Santiago: Universitaria.

Martuccelli, D. (1999). Sociologies de la modernité. Paris: Gallimard.

Núñez, I. (1997). Historia reciente de la educación chilena. Disponible en: http://historiaeducacion.tripod.com/sitebuildercontent/sitebuilderfiles/historia_reciente.doc revisado el 15 de julio de 2011.

Parsons, T. (1959). The School Class as a Social System: Some of its functions in american society. Harvard Educational Review, Vol 29, No 4, 297-318.

Parsons, T. (1990). El sistema social. Madrid: Alianza Editorial.

PIIE (1983). Las transformaciones educaciones del Régimen Militar. Santiago: PIIE.

Sarmiento, D. F. (1849). De la Educación Popular, Santiago: Julio Belin y Compañía.

Serrano, S. (1994). Universidad y Nación. Chile en el siglo XIX. Santiago: Universitaria.

Schultz, T. W. (1963). The Economic Value of Education. New York: Columbia University Press. 